\title{
CARGA DEL CUIDADO EN CUIDADORES FAMILIARES DE PERSONAS CON ENFERMEDAD CRÓNICA EN LA REGIÓN PACÍFICA COLOMBIANA
}

\section{THE BURDEN OF CARE IN FAMILY CAREGIVERS OF PATIENTS WITH CHRONIC ILLNESS IN THE COLOMBIAN PACIFIC COAST}

\author{
Gloria Mabel Carrillo* \\ Lorena Chaparro Díaz ${ }^{* *}$ \\ BeATriz SÁnchez Herrera ${ }^{* * *}$
}

\begin{abstract}
RESUMEN
Objetivo: Establecer la percepción de carga y las características sociodemográficas de un grupo de cuidadores familiares de personas con enfermedad crónica que habita en el Pacífico Colombiano. Material y método: Estudio descriptivo de corte transversal desarrollado en el Municipio de Tumaco entre los meses de agosto y noviembre de 2012 con un grupo de 55 cuidadores familiares de personas con enfermedad crónica. Resultados: El grupo de cuidadores familiares fueron en su mayoría mujeres, en edad productiva, $29 \%$ menores de 18 o mayores de 60 años, $67 \%$ menores o de la misma edad de la persona enferma; con elevado nivel de analfabetismo; $62 \%$ no finalizaron la educación primaria; $98 \%$ pertenece al estrato socioeconómico más bajo; $57 \%$ del grupo se dedicaba a las labores del hogar y $13 \%$ cumplía actividades de cuidado mientras estudiaba. Se identificó una percepción de sobrecarga intensa en el $71 \%$ de los cuidadores, leve en el 24,5\% y no sobrecarga en el 3,5\%. Conclusiones: Los resultados del estudio permiten comprender la difícil situación de cuidado de las personas con enfermedad crónica en el Pacífico colombiano, en donde a partir de la caracterización de este grupo se establece que el nivel de carga es alto en una importante proporción. Se hace un llamado urgente al Sistema de Salud Colombiano para revisar mecanismos de apoyo para las personas con ECNT y sus cuidadores familiares.
\end{abstract}

Palabras clave: Cuidadores, familia, costo de enfermedad, enfermería en salud pública.

\begin{abstract}
Objective: To characterize and define the care burden perception of family caregivers of people with chronic illness who reside in the Colombian Pacific. Methods: This is a descriptive cross section study developed in the Municipality of Tumaco, in the Colombian Pacific Region, from August to November of 2012 with a group of 55 family caregivers of people with chronic illness. Results: The family caregivers of chronic ill people are mainly women, in a productive age, $29 \%$ are younger than 18 years or older than 60 years, $67 \%$ are younger or the same age than care receptor; with a high level of illiteracy; $62 \%$ did not finished their primary school; $98 \%$ of the caregivers belong to the lowest poverty social level; $57 \%$ of the group is devoted to home activities, and $13 \%$ are

\footnotetext{
* Enfermera, Mg Enfermería, Profesora Asociada Universidad Nacional de Colombia. Facultad de Enfermería. Email: gmcarrillog@unal.edu.co. Grupo de Cuidado al paciente crónico y la familia. Calle 44 № 45-67 Bloque B2, Oficina 801. PBX 57(1) 3165000 ext.: 10340-10332-10326. Bogotá Colombia.

${ }^{* *}$ Enfermera, PhD Enfermería, Profesora Asociada Universidad Nacional de Colombia. Email: olchaparrod@unal.edu.co. Grupo de cuidado al paciente crónico y la familia.

*** Enfermera MScN, GNP. Profesora Titular Universidad Nacional de Colombia. Email: cbsanchezh@unal.edu.co. Grupo de cuidado al paciente crónico y la familia.
} 
caregivers while they study. They report a high burden with care perception in $71 \%$ of the caregivers moderate care burden in $24,5 \%$ and no care burden in 3,5\% of the cases. Conclusions: The results of the study allow understand the hard situation of well-taken care of the people with chronic disease in the Colombian Pacific in where from the characterization of this group, it settles down that the load level is high in an important proportion. An urgent call to the Colombian system of health is made to review mechanisms of support for the people with chronic illness and their family caregivers.

Key words: Caregivers, family, cost of illness, public health nursing.

Fecha recepción: 22/02/13. Fecha aceptación: 05/05/14.

\section{INTRODUCCIÓN}

La región pacífica colombiana está compuesta por cinco departamentos: Chocó, Valle del Cauca, Nariño, Cauca y una pequeña parte de Antioquia, sumando 56 municipios en total en una extensión de tierra de 113.000 $\mathrm{km}^{2}$. Se caracteriza por tener una gran riqueza cultural y biodiversa y es un punto estratégico para el país y para la inserción de su economía a nivel mundial (1-5).

La población en la región Pacifica se encuentra cobijada bajo el Sistema General de Seguridad Social en Salud (SGSSS), sin embargo el 30\% de la población no tiene cobertura por parte de ninguno de los regímenes de salud. El régimen subsidiado ofrece la mayor cobertura con el $38.8 \%$ y el contributivo el $14.1 \%$ (6). Está claramente demostrada la insuficiencia de servicios de salud de algunas regiones frente a los importantes riesgos que enfrenta (7). Las principales dificultades para acudir a un servicio de salud son las grandes distancias que se deben recorrer, las creencias étnicas y los costos asociados a acudir a un centro hospitalario.

A pesar de las condiciones propias del subdesarrollo, el perfil epidemiológico de la región Pacífica de Colombia señala paradójicamente que las Enfermedades Crónicas no Transmisibles (ECNT) ocupan las primeras causas de morbi-mortalidad y tienen una fuerte tendencia al aumento. Datos provenientes del Departamento de Nariño señalan que las defunciones por ECNT fluctúan en- tre 43 y 56 por cada 100 muertos en comparación con otras causas y que las enfermedades crónicas causaron del 30 al 36\% de la mortalidad total entre el decenio de 1998 al 2008 (8). Cinco de las seis primeras causas de muerte reportadas para el Departamento de Nariño incluyeron enfermedades crónicas, entre las que están enfermedades isquémicas del corazón, las enfermedades cerebro-vasculares, la Hipertensión arterial, las enfermedades respiratorias crónicas y la diabetes mellitus. Sin embargo, los datos de los departamentos que conforman el Pacífico colombiano no tienen desagregada la información entre las regiones Andina y la Pacífica y las propuestas departamentales se focalizan en la Región Andina (9). No se conocen estudios sobre las condiciones de los pacientes y las de sus cuidadores familiares, ni ha sido reportada la percepción de carga del cuidado de las personas con ECNT en esta región.

Ahora bien, La ECNT se caracteriza por su larga permanencia en el tiempo, no tener una causa identificada, por estar asociada con el estilo de vida y por la necesidad de cuidado que genera. La mayor parte de las personas con ECNT son cuidadas por sus familias y sus cuidadores familiares, es decir a una persona con vínculo de parentesco o cercanía que asume las responsabilidades del cuidado de un ser querido que vive en situación de ECNT. Este cuidador participa en la toma de decisiones, realiza o supervisa las actividades de la vida diaria en las cuales apoya al receptor del cuidado. El cuidador familiar requiere habilidad para dar un cuidado competente, 
sin embargo se ha encontrado que en muchos casos los cuidadores familiares carecen de la habilidad requerida y a pesar de ello deben asumir la responsabilidad $(10,11)$.

La carga del cuidado para el cuidador familiar es un fenómeno que no puede ser directamente observable o demostrable pero sí inferible. A pesar de que su esclarecimiento ha sido difícil y controvertido, se tienen avances importantes a nivel mundial logrados a través de procesos de categorización y medición de la carga del cuidado, que incluyen entre otros la percepción, la conducta o el impacto que esta carga genera $(10,12,13)$.

Enfermería como agente activo del equipo de salud está llamada a identificar las características particulares del contexto de los cuidadores de personas con ECNT, y a reconocer cómo estas características pueden incidir en fenómenos relacionados con la atención de los usuarios. Estos diagnósticos se constituyen en referentes clave para consolidar propuestas de intervención acordes con estas realidades, que muy probablemente incidirán en la disminución de la carga generada.

Este trabajo buscó establecer la percepción de carga y las características sociodemográficas de un grupo de cuidadores familiares de personas con enfermedad crónica que habita en el Pacífico Colombiano.

\section{MATERIAL Y MÉTODO}

El estudio es parte del Programa de investigación Disminución de la carga de la ECNT en Colombia ${ }^{1}$. Se desarrolló con un abordaje cuantitativo descriptivo de corte transversal, en el Municipio de Tumaco, Nariño, a través de una muestra intencional con 55 cuidadores

\footnotetext{
${ }^{1}$ Grupo de trabajo de la Unión Temporal Carga de la Enfermedad crónica No Transmisible en Colombia. Programa de Investigación: Disminución de la Carga de la Enfermedad crónica No Transmisible en Colombia. Universidad Nacional de Colombia. Financiado por Colciencias, Contrato 366 de 2011.
}

familiares de personas con ECNT. La muestra se tomó de manera intencional a través de las instituciones de salud del SGSSS, durante el segundo semestre del año 2012. Para la recolección de la información se emplearon dos instrumentos: 1) el GCC-UN C, una ficha de caracterización para los cuidadores familiares de personas con ECNT de autoría del Grupo de Cuidado al Paciente crónico de la Universidad Nacional de Colombia que incluye datos sociodemográficos como sexo, edad, estrato, relación con el cuidador, tiempo que lleva con la enfermedad crónica, nivel de religiosidad, escolaridad, empleo de TIC's, estado de salud y funcionalidad del cuidador y de la persona a quien cuida. 2) Para valorar la percepción de carga del cuidado se utilizó el instrumento Encuesta de Carga del cuidado de Zarit (14), la cual logra medir en forma multidimensional el nivel de carga que el cuidador percibe con su responsabilidad de cuidar a la persona con ECNT. Esta encuesta consta de 22 preguntas que son respondidas con una escala tipo Likert de 5 opciones que van de nunca a casi siempre. La puntuación va de 0 a 88 puntos y se interpreta de la siguiente manera: Ausencia de Sobrecarga $(<0$ igual a 46), Sobrecarga Ligera (47 a 55), Sobrecarga Intensa (> o igual a 56). La encuesta de Zarit cuenta con pruebas psicométricas en diferentes idiomas, incluido el español en donde se reporta una validez de 0.92 y una confiabilidad (consistencia interna) con un alfa de Cronbach de 0.87 (15).

Para la recolección de la información se contó con una auxiliar de investigación que vive en la zona, previamente capacitada y quien recibió acompañamiento permanente. El tiempo de las entrevistas fluctuó entre 45 y 90 min y éstas se realizaron en la institución hospitalaria o en la residencia de los participantes a su conveniencia. Los desplazamientos para realizar la entrevista duraron entre 10 min y $3 \mathrm{~h}$. Los datos fueron registrados en formato físico, se tabularon y analizaron mediante el programa estadístico SPSS en su versión 2012. 
Esta investigación mantuvo rigor ético y tuvo en cuenta la Resolución 8430 de 1993 del Ministerio de Salud de Colombia, lo que incluyó entre otras el consentimiento informado de cada participante y el manejo confidencial de la información. El Proyecto contó con aval del Comité de Ética de la Facultad de Enfermería de la Universidad Nacional de Colombia y del Consejo Directivo del Instituto de Estudios del Pacífico de la misma institución.

\section{RESULTADOS}

En la caracterización de los cuidadores de personas con enfermedad crónica no trasmisible se encontró que el $76 \%$ era de género femenino. Respecto de la edad 38\% tenía entre 36 a 59 años, 33\% de 18 a 35, 18\% mayores de 60 años y 11\% menores de 18 años. La edad de los pacientes fue menor que la del cuidador en un $62 \%$, mayor que la de éste en un $33 \%$ e igual en un $5 \%$.

El $80 \%$ de estos cuidadores sabía leer y escribir, $62 \%$ refirió escolaridad primaria incompleta, $16 \%$ bachillerato completo, 15\% bachillerato incompleto, estudios superiores $5 \%$ técnico. Pertenecían en el $98 \%$ al estrato socioeconómico 1, considerado el más bajo con un ingreso per cápita de menos de 4 dólares al día. La ocupación fue labores del hogar en un $57 \%$, trabajo independiente $20 \%$, estudiantes $13 \%$ y otros el 10\%.

Referente al estado civil, el 45\% de los cuidadores era solteros, $50 \%$ en unión libre, $5 \%$ casados. En cuanto a la orientación religiosa, el $69 \%$ refirió ser católico, $25 \%$ cristiano y $4 \%$ agnóstico. El nivel de compromiso religioso fue en un $64 \%$ bajo, $20 \%$ medio y alto en un $16 \%$.

Los cuidadores desempeñan la labor de cuidado en un $73 \%$ desde el momento del diagnóstico en el paciente y eran cuidadores únicos en el 80\% de los casos; de éstos el $42 \%$ llevaba como cuidador de 7 a 18 meses, $42 \%$ de 0 a 6 meses, $12 \%$ de 19 a 36 meses y 4\% más de 36 meses. Su relación con la persona cuidada era: $40 \%$ padres, $24 \%$ esposos, $16 \%$ hijos y $20 \%$ otros. El número de horas que estas personas dedicaban diariamente al cuidado era: $55 \%$ menos de 6 h, $27 \%$ de 7 a 12 h, $13 \% 24$ h y $5 \%$ de 13 a 23 h. El 51\% de los cuidadores no contaban con apoyo de algún tipo y el $49 \%$ refirió que contaba con apoyo de tipo social.

En cuanto al conocimiento de las Tecnologías de la Información y la Comunicación (TIC's) los cuidadores reportaron un nivel medio en el 51\% de los casos, bajo en el 38\% y alto en el restante $11 \%$; la posibilidad de acceso a estos medios es en un 58\% media, 35\% baja y $7 \%$ alta.

Los principales diagnósticos médicos encontrados en estos cuidadores familiares fueron: hipertensión arterial $22 \%$, enfermedades osteoarticulares $7 \%$, gastritis $7 \%$, asma $5 \%$, sin ningún tipo de enfermedad 53\%.

\section{Nivel de carga con el cuidado de la persona con ECNT que percibe el cuidador}

Se registró con la Encuesta de Carga del cuidado de Zarit una puntuación mínima de 39 y máxima de 86 puntos; promedio de 60.5 (DE 8.66), mediana de 61, datos que permiten visibilizar la sobrecarga global de los cuidadores familiares.

Los resultados de la aplicación de este instrumento de valoración permitieron evidenciar una sobrecarga intensa en 41 de los 55 cuidadores, representando el $74 \%$ del total; una sobrecarga leve en 12 cuidadores y no sobrecarga en el rol del cuidador en 2 personas (Gráfico 1): 
Gráfico 1. Sobrecarga de cuidadores familiares de personas con enfermedad crónica en la región Pacífico Colombiana. 2012.

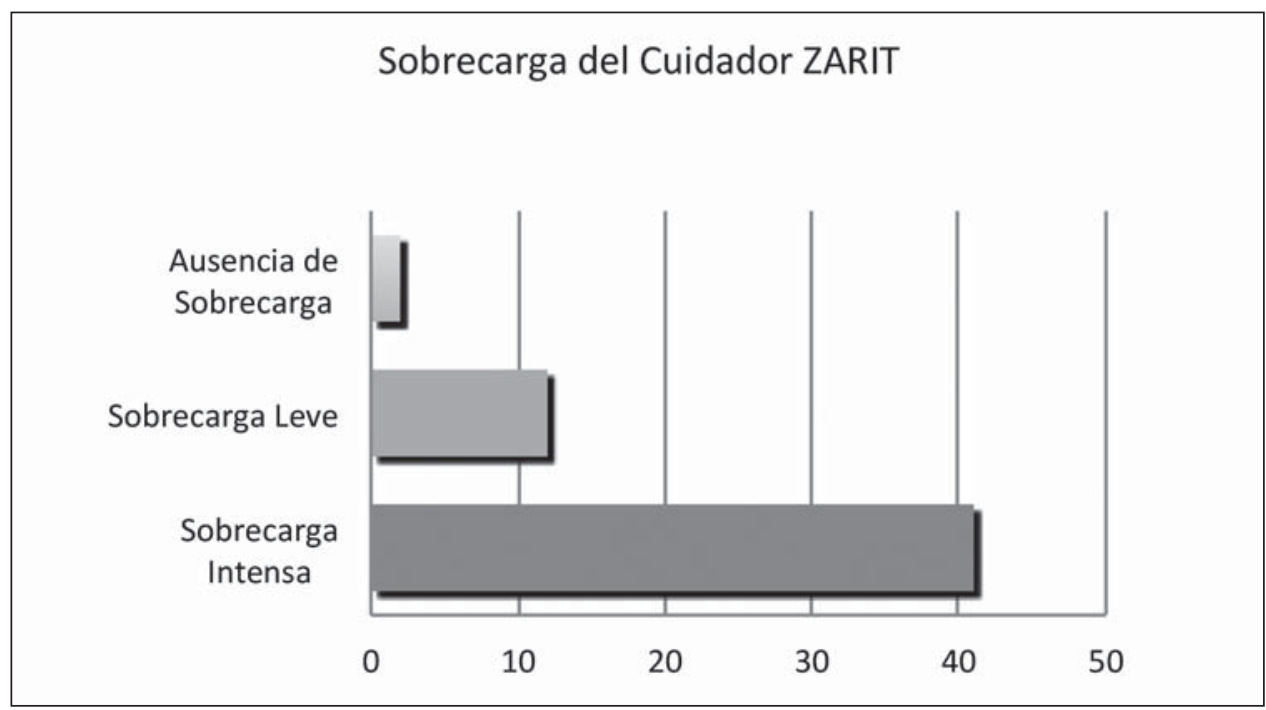

\section{DISCUSIÓN}

Este grupo de cuidadores familiares de personas con ECNT en su mayoría femenino es similar en la composición de género a lo reportado en diferentes regiones del país y en el mundo (16-20).

Con respecto a la edad llama la atención que el $29 \%$ de los cuidadores son menores de 18 o mayores de 60 , edades a las cuales responden por sus familiares enfermos en lugar de recibir el apoyo socialmente esperado. Por otra parte, la mayoría de los cuidadores son personas en edad productiva, a quienes posiblemente esta situación les afecta su plan de vida. Puede observarse en los mismos hallazgos cómo más de la mitad del grupo se dedica al hogar y un $13 \%$ cumple actividades de cuidado mientras estudia.

Es también llamativo que estos cuidadores sean menores o de la misma edad de la persona con ECNT en el $67 \%$ de los casos. Esta característica ha demostrado afectar de manera importante la capacidad del cuidador familiar para asumir su responsabilidad (21).
El elevado nivel de analfabetismo dentro de un grupo en el cual más de la mitad no finalizaron la educación primaria, sumado al hecho de tener $98 \%$ de cuidadores pertenecientes al estrato socioeconómico más bajo, deja ver una vez más las condiciones de pobreza de la región del Pacífico colombiano. Es fácil suponer que con tan baja escolaridad se tiene dificultad para dar un cuidado competente como se ha documentado en varios cuidadores de América Latina (10, 11, $16,22)$. Se suman a ello las pocas posibilidades de beneficiarse de sistemas modernos de apoyo a los cuidadores familiares como los que brindan las TIC's y que han sido documentados en pacientes fumadores, diabéticos y en cuidadores de personas con ECNT (23-26). Esta situación elimina posibilidades que podrían contribuir a la equidad dentro del SGSSS (27). De hecho, solo 11\% de estos cuidadores refieren tener buen nivel de conocimiento sobre las TIC's y un porcentaje mínimo acceso adecuado a las mismas.

Estos cuidadores cuentan con un compañero permanente, en la mitad de los casos, lo que puede constituirse en un factor protector. Sin embargo, a partir de la información 
del tipo de vínculo con la persona cuidada, el $24 \%$ cuida a su pareja y en tal caso, el soporte social puede no ser igualmente efectivo.

Es posible además que haya cansancio por cuanto el 73\% de los cuidadores estén al frente de su responsabilidad desde que apareció la situación de ECNT y que se identifiquen como cuidadores únicos en $80 \%$ de los casos. Casi la mitad reporta tener que atender a su familiar por más de 7 horas al día y la mitad refieren no tener ningún tipo de apoyo.

La espiritualidad en general y la religión, en particular, han sido señaladas como dimensiones del bienestar que apoyan la calidad de vida en la experiencia de la ECNT (28). Sin embargo, si bien el 96\% de los cuidadores señalan tener un vínculo con alguna religión, llama la atención que solo el 16\% menciona su compromiso religioso como algo importante.

Desde el punto de vista de la salud pública, quizá el dato más alarmante es que más de la mitad (53\%) de los cuidadores familiares refieren enfermedades típicamente asociadas al estrés, muchas de ellas también de carácter crónico. Este hallazgo concuerda con estudios que muestran disminución en la calidad de vida y la salud de los cuidadores $(29,30)$.

Con respecto a la percepción de la carga del cuidado de las personas con ECNT se evidencia sobrecarga en el $96,5 \%$ de los cuidadores, siendo intensa en el $74 \%$ de ellos, lo que revisando el contexto y las características socioeconómicas no resulta sorprendente, pero sí muy preocupante. Hallazgos similares de sobrecarga presentan diferentes estudios (31-34) aunque su reporte no es tan dramático como el que mostró este grupo que supera en mucho las condiciones de dificultad y el nivel de sobrecarga percibido por otros grupos de cuidadores.

El contexto/ambiente puede incidir directamente en la percepción de la carga del cuidado. Al respecto, Robinson (35) resalta que el sueño, el tiempo requerido, el esfuerzo físico, la confinación, los ajustes familiares y emocionales, el cambio de planes, la demanda de tiempo, el comportamiento disruptivo, la carga económica y la sensación de desbordamiento, entre otras, son variables que ligadas al ambiente en que se ofrece la atención, afectan el índice de carga del cuidado en el cuidador.

En consecuencia con lo anterior, se infiere que las consecuencias adversas que se presentarán en los cuidadores tendrán impacto devastador sobre su salud física y emocional, así como mayores costos económicos poco reconocidos y estudiados en un contexto de alta vulnerabilidad dado por las variables sociodemográficas que lo caracterizan.

La caracterización y definición de la percepción de carga del cuidado que tiene un grupo de cuidadores familiares de personas con enfermedad crónica que habita en el Municipio de Tumaco, en el Pacífico Colombiano permite visibilizar por una parte las difíciles condiciones en que esta población vive, por otra las dificultades que tiene para asumir responsabilidades de cuidado de las personas con ECNT y que son percibidas por ellos como una muy elevada carga de cuidado y por último la precaria situación de un SGSSS de la región (3) que no ha definido estrategias para la atención de las personas con ECNT ni para sus cuidadores, quienes se han convertido, como sucede en más de la mitad de los casos, en pacientes con ECNT.

Para algunas instancias del SGSSS puede ser justificable por ser zonas de alto conflicto de fuerzas armadas; sin embargo, con la presencia nacional de instituciones de educación como la Universidad Nacional de Colombia y su infraestructura tecnológica, se debe aprovechar más la telepresencia con formación del personal de la salud en estrategias que aborden la disminución de la carga de la ECNT. Por este primer elemento de análisis en el marco de un programa de investigación, los resultados de la primera fase del proyecto arrojarán resultados importantes para la enfermería y la salud pública. 


\section{REFERENCIAS}

1. Martínez A. Entre la diversidad y la desigualdad: diagnóstico territorial del Pacífico colombiano, en perspectivas de derechos humanos. Bogotá: Editorial Kimpres Ltda. 2010.

2. Montoya I, Montoya L, Muñoz G, Sánchez B. Fronteras. Elementos estratégicos para una visión de las fronteras desde la Universidad Nacional de Colombia. Bogotá: Editorial Universidad Nacional de Colombia. 2012. p. 125-136.

3. Departamento Administrativo Nacional de Estadística DANE. Estadísticas vitales: Censo demográfico del 2005. [Internet]. Bogotá: DANE; 2005 [actualizado 30 noviembre 2012; citado 5 de diciembre 2012]. Disponible en: http://www.DANE. gov.co/DANEweb_V09

4. Universidad Nacional de Colombia. La educación, una estrategia para el avance del Pacífico colombiano. [Propuesta] Bogotá: Universidad Nacional de Colombia; 2011. p. 13-15.

5. Grueso L. Representaciones y relaciones en la construcción del proyecto político y cultural del Proceso de Comunidades Negras en el contexto del conflicto armado en la región del Pacífico Sur colombiano. En: Mato D, editores. Políticas de economía, ambiente y sociedad en tiempos de globalización. Caracas: Facultad de Ciencias Económicas y Sociales; 2005. p. 53-70.

6. Martínez A. Entre la diversidad y la desigualdad: diagnóstico territorial del Pacífico colombiano, en perspectivas de derechos humanos. Bogotá: Editorial Kimpres Ltda; 2010. p. 30-31.

7. Agudelo O. Informe de Gestión Sede Tumaco, Universidad Nacional de Colombia. [Internet]. Bogotá: Universidad Nacional de Colombia; 2011 [citado 6 octubre 2012] Disponible en: http://www. tumacopacifico.unal.edu.co
8. Asociación Internacional de Institutos de Salud Pública, Ministerio de la Protección Social, Instituto Nacional de Salud, Alcaldía de Pasto, Secretaría Municipal de Salud. Observatorio de enfermedades crónicas. Boletín No 1 Evidencia de las enfermedades crónicas para la toma de decisiones en Pasto. Serie de informes técnicos. San Juan de Pasto: Observatorio de Enfermedades Crónicas; 2011.

9. Lucumí D, Gutiérrez A, Moreno J, Gómez L, Lagos N, Rosero M, et al. Planeación local para enfrentar el desafío de las enfermedades crónicas en Pasto, Colombia. Rev Salud Publica. 2008; 10(2): 343-351.

10. Barrera OL, Pinto AN, Sánchez HB, Galvis $C$, Moreno $M$, Pinzón $M$, et al. La habilidad de cuidado de los cuidadores familiares de personas con enfermedad crónica: Un estudio comparativo en tres unidades académicas de enfermería. Invest educ enferm. 2006; 24(1): 36-46.

11. Pinto N, Barrera L, Sánchez B, Figueroa P, De Camargo L. Habilidad de cuidado de los cuidadores familiares de personas con enfermedad crónica: Mirada internacional. Aquichan. 2006; 6(1): 22-33.

12. Sánchez HB, Carrillo GM, Barrera OL, Chaparro DL. Carga del cuidado de la enfermedad crónica no transmisible. Aquichan 2013; 13(2): 247-260.

13. Sánchez HB, Carrillo GM, Chaparro DL. Escalas de medición de carga de la enfermedad crónica. Actual. enferm. En prensa 2014.

14. Zarit SH, Reever KE, Bach-Peterson J. Relatives of the impaired elderly: correlates of feelings of burden. Gerontologist 1980; 20: 649-55.

15. Breinbauer H, Vásquez H, Mayanz S, Guerra C, Millán T. Validación en Chile de la Escala de Sobrecarga del Cuidador de Zarit en sus versiones original y abreviada. Rev Med Chil [Internet]. 2009 [citado 7 octubre 2012]; 137(1): 657-665. Disponible en: http://www.scielo.cl/pdf/ rmc/v137n5/art09.pdf 
16. Barrera L, Pinto N, Sánchez B. Habilidad de cuidado de cuidadores familiares de personas con enfermedad crónica: Comparación de géneros. Actual. enferm. 2006; 9(2): 9-13.

17. Marks NF, Lambert JD, Choi H. Transitions to Caregiving, Gender, and Psychological Well-Being: A Prospective U.S. National Study. J Marriage Fam. 2002; 64: 657-667.

18. Navaie-Waliser M, Spriggs A, Feldman $\mathrm{PH}$. Informal caregiving differential experiences by Gender. Rev Medical Care 2002; 40(12): 1249-1259.

19. Vaquiro S, Stiepovich J. Cuidado informal, un reto asumido por la mujer. Cienc enferm 2010; XVI(2): 17-24.

20. Maume DJ. Gender Differences in Restricting Work Efforts Because of Family Responsibilities. J Marriage Fam. 2006; 68: 859-869.

21. Luengo Martínez C, Araneda Pagliotti G, López Espinoza M. Factores del cuidador familiar que influyen en el cumplimiento de los cuidados básicos del usuario postrado. Index Enferm. 2010; 19(1): 14-18.

22. Barrera L, Pinto N, Sánchez B. Evaluación de un programa para fortalecer a los cuidadores familiares de enfermos crónicos. Rev Salud Publica. 2006; 8(2): 141-152.

23. Del Pozo J, Ferreras M. Adicciones y Nuevas Tecnologías de la Información y de la Comunicación: Perspectivas de su uso para la prevención y el tratamiento. Dirección General de Salud Pública y Consumo Servicio de Drogodependencias Gobierno de La Rioja, España: Impresiones Quintana; 2009.

24. Curioso W, Gozzer E, Valderrama M, Rodríguez-Abad J, Villena J, Villena A. Uso y percepciones hacia las tecnologías de información y comunicación en pacientes con diabetes en un hospital público del Perú. Rev Peru Med Exp Salud Pública. 2009; 26(2): 161-67.

25. Barrera L, Carrillo G, Chaparro L, Pinto N, Sánchez B. Soporte social con el uso de las TIC's para cuidadores familiares de personas con enfermedad crónica. Rev Salud Pública. 2011; 13(3): 446-57.

26. Carrillo G, Chaparro Díaz L, Barrera Ortiz L, Pinto Afanador N, Sánchez Herrera B. El Blog como Herramienta de Soporte Social para Personas con Enfermedad Crónica. Cienc enferm. 2011; XVII(3): 137-149.

27. Jardines Méndez JB. Acceso a la información y equidad en salud. Rev Cub Salud Pública [Internet]. 2007 [citado 5 diciembre 2012]; 33(3). Disponible en: http://scielo.sld.cu

28. Sánchez B. Dimensión espiritual del cuidado en situaciones de cronicidad y muerte. [Internet]. Bogotá: Editorial Universidad Nacional de Colombia; 2004 [citado 3 noviembre 2012]. 288 p. Disponible en: http://www.bdigital.unal.edu. co/2050/

29. García M, Mateo I, Maroto G. El impacto de cuidar en la salud y la calidad de vida de las mujeres. Gac Sanit. 2004; 18(2): 84 $-87$.

30. López M, Orueta R, Gómez S, Sánchez A. Carmona de la Morena J., Alonso Moreno F. El rol de Cuidador de personas dependientes y sus repercusiones sobre su Calidad de Vida y su Salud. Rev Clin Med Fam. 2009; 2(7): 332-339.

31. González A, Gálvez C. Características sociodemográficas de salud y utilización de recursos sanitarios de cuidadores de ancianos atendidos en domicilio. Gerokomos [Internet]. 2009 [citado 3 noviembre 2012]; 20(1): 16-21. Disponible en: http:// scielo.isciii.es/pdf/geroko/v20n1/15rincon.pdf

32. Flores E, Rivas E, Seguel F. Nivel de sobrecarga en el desempeño del rol del cuidador familiar de adulto mayor con dependencia severa. Cienc enferm. 2012; XVIII(1): 29-41.

33. Flórez I., Montalvo A, Herrera A, Romero E. Afectación de los bienestares en cuidadores de niños y adultos con enfermedad 
crónica. Rev Salud Pública [Internet]. 2010 [citado 5 noviembre 2012]; 12(5): 755-763. Disponible en: http://www.scielo.org.co/pdf/rsap/v12n5/v12n5a06.pdf

34. Espinoza K, Jofre V. Sobrecarga, Apoyo Social y Autocuidado en Cuidadores In- formales. Cienc enferm. 2012; XVIII(2): 23-30.

35. Robinson BC. Validation of caregiver Strain Index. J Gerontol. 1983; 38(3): 344-348. 